\title{
Protease activity at invadopodial focal digestive areas is dependent on NHE1-driven acidic pHe
}

\author{
MARIA RAFFAELLA GRECO ${ }^{1}$, ESTER ANTELMI ${ }^{1,2}$, GIOVANNI BUSCO $^{1}$, LORENZO GUERRA ${ }^{1}$, \\ ROSA RUBINO $^{1}$, VALERIA CASAVOLA ${ }^{1}$, STEPHAN JOEL RESHKIN $^{1 *}$ and ROSA ANGELA CARDONE ${ }^{1 *}$ \\ ${ }^{1}$ Department of Biosciences, Biotechnology and Biopharmaceutics, University of Bari, I-70126 Bari, Italy
}

Received October 7, 2013; Accepted November 29, 2013

DOI: 10.3892/or.2013.2923

\begin{abstract}
Degradation of the extracellular matrix (ECM) is a critical step of tumor cell invasion and requires proteasedependent proteolysis focalized at the invadopodia where the proteolysis of the ECM occurs. Most of the extracellular proteases belong to serine- or metallo-proteases and the invadopodia is where protease activity is regulated. While recent data looking at global protease activity in the growth medium reported that their activity and role in invasion is dependent on $\mathrm{Na}^{+} / \mathrm{H}^{+}$exchanger 1 (NHE1)-driven extracellular acidification, there is no data on this aspect at the invadopodia, and an open question remains whether this acid extracellular $\mathrm{pH}(\mathrm{pHe})$ activation of proteases in tumor cells occurs preferentially at invadopodia. We previously reported that the NHE1 is expressed in breast cancer invadopodia and that the NHE1-dependent acidification of the peri-invadopodial space is critical for ECM proteolysis. In the present study, using, for the first time, in situ zymography analysis, we demonstrated a concordance between NHE1 activity, extracellular acidification and protease activity at invadopodia to finely regulate ECM digestion. We demonstrated that: (i) ECM proteolysis taking place at invadopodia is driven by acidification of the peri-invadopodia microenvironment; (ii) that the proteases have a functional pHe optimum that is acidic; (iii) more than one protease is functioning to digest the ECM at these invadopodial sites of ECM proteolysis; and (iv) lowering $\mathrm{pHe}$ or inhibiting the NHE1 increases protease secretion while blocking protease activity changes NHE1 expression at the invadopodia.
\end{abstract}

Correspondence to: Professor Stephan Joel Reshkin, Department of Biosciences, Biotechnology and Biopharmaceutics, University of Bari, Via Amendola 165/A, I-70126 Bari, Italy

E-mail: reshkin@biologia.uniba.it

Present address: ${ }^{2}$ Department of Pathology, Anatomic Pathology A Unit, Istituto Nazionale Tumori, Milan, Italy

*Contributed equally

Key words: $\mathrm{Na}^{+} / \mathrm{H}^{+}$exchanger 1, breast cancer, protease, invadopodia, invasion, extracellular matrix proteolysis

\section{Introduction}

The tumor microenvironment plays a fundamental role in promoting tumor progression and metastasis (1). One of the primary forces of the metabolic tumor microenvironment guiding an invasive and metastatic phenotype is the low extracellular $\mathrm{pH}(\mathrm{pHe})$ produced by tumor cells $(2,3)$. Studies using in vitro cell-culture and in situ tumor spectroscopy have revealed that, while having an alkaline intracellular $\mathrm{pH}(\mathrm{pHi})$, tumors can reach an acidic interstitial pHe 0.5-1 units lower than normal tissue (tumor pHe of 6.5-7 vs. a normal tissue $\mathrm{pHe}$ of 7.4) (4). This acidic pHe was previously demonstrated to stimulate in vitro invasion (5) and in vivo metastasis (6), but the mechanism(s) underlying acidic pHe-induced effects are only recently being defined. According to the 'acid-mediate invasion hypothesis' (7), acidic conditions promote extracellular release and activity of key proteases such as cathepsin B and matrix metalloproteinase-2 and -9 (MMP-2, -9) (8), which break down both extracellular matrix (ECM) and basement membrane, thereby promoting migration, invasion and metastasis (9). Therefore, we initiated studies to elucidate the nature of the molecular pathways and mechanisms that regulate these events.

Aggressive tumor cells from multiple malignancies localize and concentrate a series of different proteases including the secreted MMP-2 and -9 and the transmembrane proteases (such as MT1-MMP) at actin-rich invasive protrusions called invadopodia that precisely regulate the directed proteolysis of the ECM and facilitate invasion (10). A wide variety of actin-interacting proteins, scaffolding proteins, signaling proteins and ion transporters are involved in invadopodia formation and functioning (11). In particular, the plasma membrane $\mathrm{Na}^{+} / \mathrm{H}^{+}$ exchanger 1 (NHE1) is localized at cancer cell invadopodia, where it plays an integrated role in both invadopodia formation and proteolytic activity (12). Taken together, these data suggest that there exists at invadopodia a concordance between NHE1 localization, extracellular acidification, protease activity on the cell surface at invadopodia and the cytoskeleton reorganization necessary for invadopodial maturation in human malignant breast carcinoma cells. There is currently no report on the effect of NHE1-induced acid pHe on protease activity at the most important sites of ECM digestion by cancer cells, the invadopodia. This is due to the fact that, although biochemical studies can determine net protease activity in the tumor extracellular medium, they do not provide any informa- 
tion if the localization and activation of proteases may occur at invadopodia. Here, by using in situ invadopodial zymography, we measured the individual activity of the different proteases and related this invadopodial-localized protease expression and activity to NHE1 and pHe at invadopodia of the human metastatic breast cancer cell line, MDA-MB-231.

\section{Materials and methods}

Reagents.Porcine skingelatin,type A, was from Sigma. Matrigel growth factor reduced w/o phenol red from BD Biosciences. $\mathrm{DQ}^{\mathrm{TM}}$ Green BSA, DQ ${ }^{\mathrm{TM}}$ Red BSA and $\mathrm{DQ}^{\mathrm{TM}}$ pig skin gelatin fluorescein conjugated from Molecular Probes. Primary antibodies: monoclonal [4E9] anti-NHE1 (Abcam), monoclonal anti-Cortactin (p80/85) clone 4F1 (Millipore), polyclonal anti-MMP-2 and anti-MMP-9 (Cell Signaling Technology, Inc.), polyclonal anti-cathepsin B (Fitzgerald), monoclonal anti- $\beta$ actin (Sigma). Rhodamine B, isothiocyanate, mixed isomers (TRITC) was from Sigma. Immunofluorescence: polyclonal anti-NHE1 (Alpha Diagnostic). Secondary antibodies: anti-mouse (Sigma) and anti-rabbit (Cell Signaling Technology, Inc.) HRP linked antibodies, goat anti-mouse and anti-rabbit Alexa Fluor 488- and Alexa Fluor 568-linked (Molecular Probes, Inc., Eugene, OR, USA).

Cell culture and preparation of different pHe in growth medium and protease inhibitors. MDA-MB-231 cells were grown in Dulbecco's modified Eagle's medium (DMEM) high glucose $(4,500 \mathrm{mg} / \mathrm{l})$ supplemented with $\mathrm{NaHCO}_{3}$ $(3,700 \mathrm{mg} / \mathrm{l}), 10 \%(\mathrm{v} / \mathrm{v})$ heat-inactivated fetal bovine serum, L-glutamine $(2 \mathrm{mM})$, sodium-pyruvate $(1 \mathrm{mg} / \mathrm{ml})$ and penicillin (100 units)/streptomycin $(100 \mathrm{mg} / \mathrm{ml})$ in a $5 \% \mathrm{CO}_{2} / 95 \%$ air humidified incubator at $37^{\circ} \mathrm{C}$.

Protease expression levels and in situ protease zymography (i.e. invadopodia-dependent ECM digestion) were measured at neutral (pHe 7.4), acidic (pHe 6.7) or basic (pHe 7.8) growth mediums, in the absence or presence of the following protease inhibitors: cathespin B inhibitor CA-074 (5 $\mu \mathrm{M})$, Calbiochem (cat. no. 205030), MMP-9 inhibitor 1 (7.5 nM) Calbiochem (cat. no. 444278) or MMP-2 inhibitor $1(15 \mu \mathrm{M})$ Calbiochem (cat. no. 444244), Batimastat (Sigma) and Cariporide (SanofiAventis).

Degradation assay and in situ protease zymography. The activity of each protease was assayed at invadopodia by measuring the quantitative levels of focal and pericellular ECM digestion via in situ zymography in cells plated on Matrigel containing quenched DQ-BSA, as previously described (12), in the absence and presence of the specific inhibitor of each protease.

Invadopodia cell fractionation. Cytosol, membrane and invadopodia fractions were obtained from cells grown on gelatin, as previously described (12).

Preparation of conditioned media, cell lysates and western blotting. These procedures were performed as previously described (13).

Image acquisition and analysis. Images were acquired and analyzed as previously described (14) with a x60 oil objective

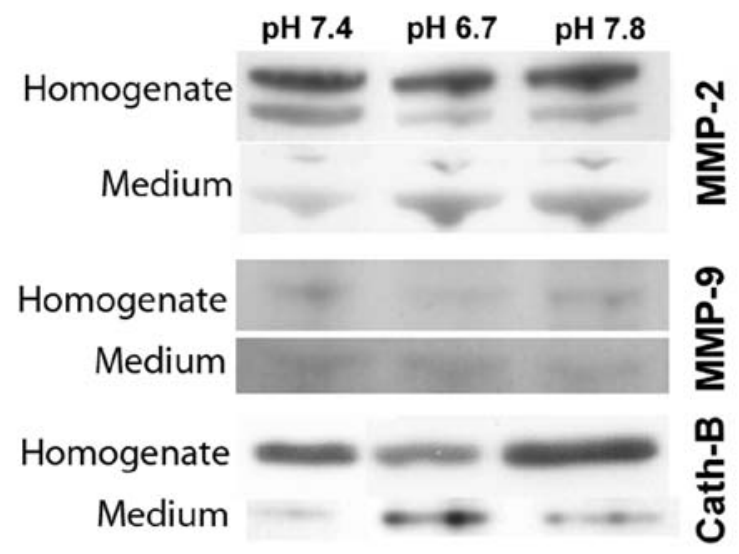

Figure 1. Stimulated release of MMP-2, -9 and cathepsin B by MDA-MB-231 cells cultured with acidic $\mathrm{pH}$ medium. MDA-MB-231 cells were cultured with control medium or medium at different $\mathrm{pH}$ and both total cell homogenates and conditioned media, after being removed and concentrated, were analyzed by western blotting with anti-MMP2, anti-MMP-9 and anti-cathepsin B antibodies. MMP-2, matrix metalloproteinase-2.

using a Nikon Eclipse TE 2000S epifluorescence microscope or a laser scanning confocal microscope (LSCM) (C1/TE2000-U; Nikon Instruments S.p.A., Sesto Fiorentino-FI, Italy). Confocal images were analyzed using ImageJ (http://rsb.info.nih.gov/ij/).

Duolink proximity ligation assay (PLA). Cells were plated for the invadopodial matrix degradation assay, fixed, permeabilized and stained with primary antibodies (NHE1, MMP-2, MMP-9 and cathepsin B) at the recommended immunofluorescence dilution (or 1:400 if no dilution was given). Proximity ligation was performed according to the manufacturer's protocol using the Duolink Detection kit with PLA PLUS and MINUS probes for mouse and rabbit (Olink Biosciences; ref. 43). Samples were analyzed with a confocal microscope (LSM 510 Meta; Carl Zeiss Inc.) under a x63 oil objective.

Statistical analysis. Student's t-test was applied to analyze the statistical significance between treatments and $\mathrm{p}<0.05$ was considered to indicate a statistically significant difference, assuming equal variances on all experimental data sets. All comparisons were performed with InStat (GraphPad Software, San Diego, CA, USA).

\section{Results}

Acidic pHe increases MMP-2, -9 and cathepsin B secretion. A correlation between tumor progression and metastasis and elevated levels of the zinc-dependent matrix metalloproteases MMP-2 and -9 (15) and/or the acidic cysteine proteases (16) has been reported in several experimental and clinical studies. Furthermore, the acidic microenvironment has been shown to be essential for promoting expression and activity of these proteases in both cancer and stromal cells during tumor invasion, angiogenesis and metastasis $(6,17)$. Therefore, we focused on MMP-2, -9 and cathepsin B to first assess how shifting the $\mathrm{pHe}$ of culture medium of breast cancer cells towards acidic or alkaline values changes their cellular levels and extracellular secretion. We incubated metastatic breast cancer cells, MDA-MB-231, with media at various $\mathrm{pH}(\mathrm{pH} 6.7, \mathrm{pH} 7.4$ and 
A

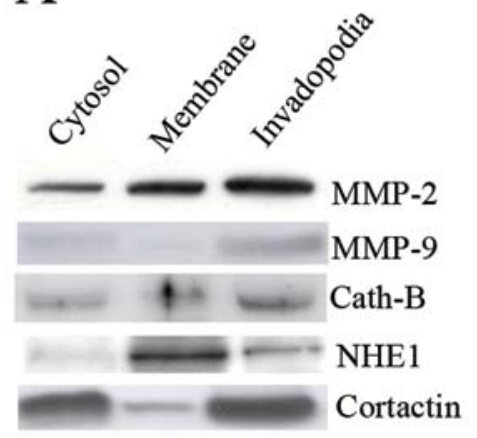

B

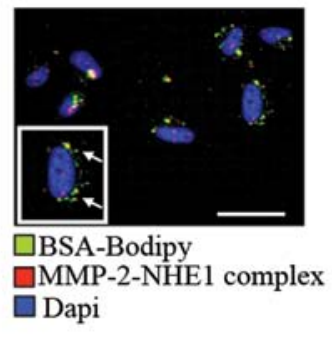

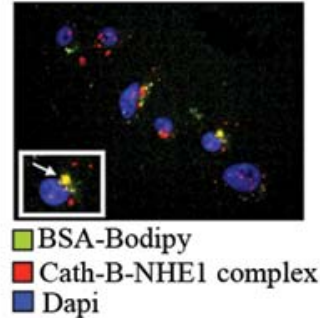

Figure 2. MMP-2/-9 and cathepsin B colocalize with NHE1 at invadopodia. (A) MMP-2/9, cathepsin B and NHE1 protein expression were assayed by western blotting in cytosolic, plasma membrane and invadopodia subcellular fractions biochemically extracted from cells incubated for $24 \mathrm{~h}$ on a thick layer of cross-linked gelatin as described in Materials and methods. The invadopodia marker, cortactin, was enriched in the invadopodia fraction. (B) Representative micrographs of NHE1 with MMP-2/-9 or cathepsin B co-localization measured with in situ PLA in cells cultured on BSA/Bodipy-Matrigel overnight. DAPI was used for nuclear staining. Scale bar, $50 \mu \mathrm{M}$. MMP-2/-9, matrix metalloproteinase-2/-9; NHE1, $\mathrm{Na}^{+} / \mathrm{H}^{+}$exchanger 1 .

$\mathrm{pH}$ 7.8) for $8 \mathrm{~h}$ and measured the expression levels of MMP-2, -9 and cathepsin $\mathrm{B}$ by western blot analysis in both the total cell homogenates and in the collected tumor conditioned medium. As seen in Fig. 1, cells incubated with acidic medium (pH 6.7) in comparison to neutral medium ( $\mathrm{pH} 7.4$ ), exhibited a decrease of the MMP-2 (72 kDa) and MMP-9 (92 kDa) inactive pro-forms in the total cell homogenates and a marked increase of their mature forms (64 and $84 \mathrm{kDa}$ respectively) released into the tumor conditioned medium. Similarly, extracellular acidosis ( $\mathrm{pH}$ 6.7) reduced cathepsin B expression in the cellular homogenate while highly inducing its secretion into the tumor extracellular medium. On the contrary, when cells were incubated with alkaline medium ( $\mathrm{pH} 7.8$ ), we found that MMP-9 and cathepsin B did not change their expression in homogenates or secretion into conditioned media when compared to cells cultured at neutral pH, while MMP-2 continued to show decreased levels in the cellular homogenates and an increased secretion into the tumor conditioned medium. The data presented here confirm that extracellular acidic conditions increase the secretion of the activated forms of MMP-2, MMP-9 and cathepsin B. However, it remains unknown whether this increased secretion occurs globally for the cell or preferentially at invadopodia, known to be centers of enrichment for a variety of proteases.

Cathepsin B, MMP-2 and -9 directly interact with NHE1 at matrix-degrading invadopodia. As we recently demonstrated that invadopodia are focal hotspots of very acidic $\mathrm{pHe}$ driven by high levels of NHE1 activity (12) and that this NHE1 activity is necessary for the digestive activity of the invadopodia, we explored whether the lowered peri-invadopodia $\mathrm{pHe}$ driving focal ECM degradation is through the regulation of the expression and/or activity of proteases at invadopodia. We first determined the presence of the three major proteases, cathepsin B, MMP-2 and -9, in invadopodia by fractionating cells plated on cross-linked porcine gelatin into cytosol, cell membrane or invadopodia fractions (12). The invadopodia compartment was identified on the basis of a strong cortactin overexpression when compared to the membrane fraction $(12,18,19)$. As can be seen in Fig. 2A, all three proteases were enriched in the invadopodia although with different rela- tive distributions. Furthermore, as previously reported (12), NHE1 was also expressed in the invadopodia compartment. To further analyze the potential association among NHE1, MMP-2, -9 and cathepsin B at proteolytically active invadopodia, a PLA, which can detect endogenous protein-protein interactions that occur within $40 \mathrm{~nm}$ (20), was combined with a Matrigel degradation assay, in which cells are plated on a mixture of Matrigel containing DQ Green BSA-Bodipy and a green fluorescent emission staining is indicative of invadopodia driven-ECM digestion (12). The advantages of PLA are that this technique provides a fluorescent signal (red) only when two target proteins are colocalized, allowing improved sensitivity for establishing endogenous protein-protein interactions and giving in situ information whether these colocalizations occur in specific intracellular compartments. As shown by the red fluorescent staining reported in Fig. 2B, NHE1 associated with all three proteases at the level of matrix-degrading invadopodia (shown in green, while the merged area in yellow are indicated with arrows). These results demonstrate that some sub-populations of MMP-2, -9 and cathepsin B may reside at the level of functionally active invadopodia where they interact with NHE1.

Protease activity suppression increases relative invadopodial NHE1 expression, while NHE1 inhibition increases acidinduced protease secretion. As both NHE1 and MMPs are involved in the invadopodia mediated-ECM degradation, and having demonstrated in Fig. 2A that breast cancer cells have an enrichment of NHE1 and protease expression at invadopodia when compared to cellular bodies, we investigated if proteases and NHE1 exert a reciprocal transmodulation of their expression and/or activities. We first evaluated the role of MMP activity on NHE1 compartmentalization in invadopodia by using a general MMP inhibitor, batimastat. As can be observed in the invadopodia fractionation experiments reported in Fig. 3A, treatment with $5 \mu \mathrm{M}$ batimastat for $6 \mathrm{~h}$ significantly redistributed NHE1 from cellular bodies to invadopodia while having no effect on the distribution of a series of other invadopodia located proteins, suggesting a feed-back role of these enzymes on invadopodia proteolytic activity in breast cancer cells by controlling NHE1 expression 

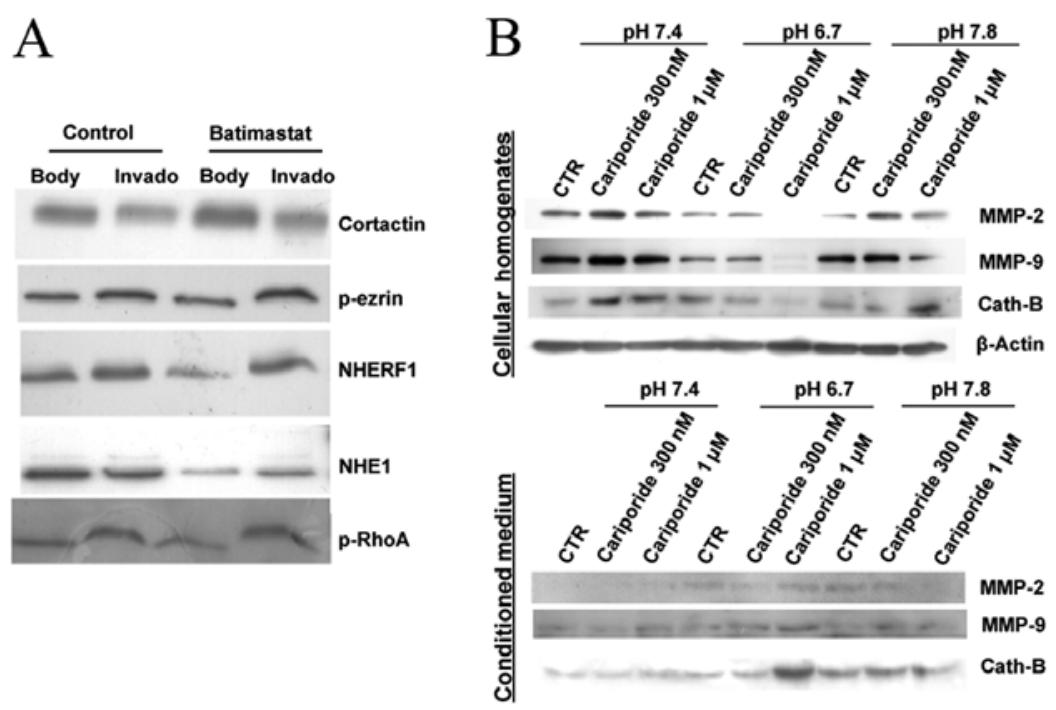

Figure 3. Inhibition of protease activity increases the relative amount of NHE1 at invadopodia while inhibition of NHE1 increases acid-induced protease secretion. (A) Immunoblotting analysis of the level of some invadopodia-residing proteins in subcellular fractions (cellular bodies and invadopodia) obtained by both control cells and cells treated for $6 \mathrm{~h}$ with the general MMP inhibitor batimastat $(5 \mu \mathrm{M})$. (B) Western blotting of MMP-2/-9, cathepsin B and NHE1 in both the cellular homogenates and the tumor conditioned medium from cells treated or not with cariporide and cultured overnight in medium at different pHe (pH 7.4, $\mathrm{pH} 6.7$ and $\mathrm{pH}$ 7.8). $\mathrm{NHE1}, \mathrm{Na}^{+} / \mathrm{H}^{+}$exchanger 1; MMP, matrix metalloproteinase; $\mathrm{pHe}$, extracellular $\mathrm{pH}$.

and localization. These data are consistent with evidence that: (i) inhibiting NHE1 activity reduced MMP-2 and -9 activation (21) and cathepsin B activation (5) in the extracellular medium, and (ii) that the overexpression of a non-transporting NHE1 mutant reduced MMP-9 expression and activity (22).

To determine whether inhibition of NHE1 by different doses of cariporide affects protease release and if there is a $\mathrm{pH}$-dependence of this process, we examined the effect of cariporide on MMP-2, -9 and cathepsin B expression at the same $\mathrm{pHe}$ values utilized in the previous experiments shown in Fig. 1. As shown in Fig. 3B, when NHE1 activity was inhibited by $300 \mathrm{nM}$ cariporide in cells grown at physiological $\mathrm{pH}(\mathrm{pH}$ 7.4), we observed an intracellular accumulation of the three proteases and a corresponding reduction of their secretion in the tumor conditioned medium. As previously, we found that acidic pHe increased the release of MMP-2, -9 and cathepsin B and inhibition of NHE1 by cariporide (especially at $1 \mu \mathrm{M}$ ) strongly stimulated the release of the proteases almost completely emptying the cells of all three proteases resulting in peaks of their levels in the conditioned medium. The use of cariporide concentrations near its $\mathrm{IC}_{50}$ value $(\sim 280 \mathrm{nM})$ and at a near maximal concentration $(1 \mu \mathrm{M})$ for inhibiting NHE1 activity (12), demonstrated that the release of the proteases have somewhat different interaction kinetics with NHE1 activity.

Acidic pHe-dependent protease activity is localized to the invadopodia. We finally determined the activity and role of the three proteases at the level of invadopodia. Similar to studies of experimental extracellular acidification in determining osteoclastic proteolytic programs $(23,24)$, we examined whether exposure to an experimental acidosis may increase focal, invadopodia-dependent ECM degradation in MDA-MB-231 cells plated on Matrigel containing DQ Green BSA-Bodipy and if this increase was due, at least in part, to a direct increase in protease activity. To determine the $\mathrm{pH}$-dependence of the activity of the specific proteases MMP-2, -9 and cathepsin B at the invadopodia, we performed focal digestion experiments via in situ zymography in Matrigel by incubating the cells in acidic (pHe 6.7), neutral (pHe 7.4) or basic (pHe 7.8) growth mediums, in the absence or presence of the following small-molecule inhibitors to pharmacologically knock-out protease function: cathepsin B inhibitor CA-074 $(5 \mu \mathrm{M})$, MMP-9 inhibitor 1 (7.5 nM) or MMP-2 inhibitor $1(15 \mu \mathrm{M})$. Proteolysis of individual cells was followed as previously described (12) with invadopodia-dependent proteolysis being defined as the pixel density of focal zones of digestion for each cell. Fig. 4A shows confocal immunofluorescence images, using this in situ zymographic assay, of the actin cytoskeleton (red) and digestion (green) from typical cells of the different pHe treatments, while Fig. 4B shows the histogram of the cumulative data of total invadopodia (focal) ECM proteolysis from four independent experiments. Exposure to extracellular acidification produced a significant increase in both invadopodia expression/number (percent of proteolytically active cells, data not shown) and total invadopodial activity in control cells (red bars in Fig. 4B) when compared to cells treated at neutral or basic $\mathrm{pHe}$. These results confirm data from previous studies showing the $\mathrm{pH}$ dependence of ECM digestion at invadopodia (12). Notably, the ability of all three protease inhibitors to block the invadopodia-localized proteolysis was much greater in the acidic medium and again, declined with increasing pHe demonstrating that, indeed, MMP-2 (green bars), MMP-9 (blue bars) and cathepsin B (yellow bars) are more active at acidic pHe.

Collectively, these data show that, as in osteoclasts, an experimental acidification increases tumor cell proteolytic activity by turning on intrinsic invadopodial programs and that an important future direction will be to identify the molecular components of these specific invasive programs. 

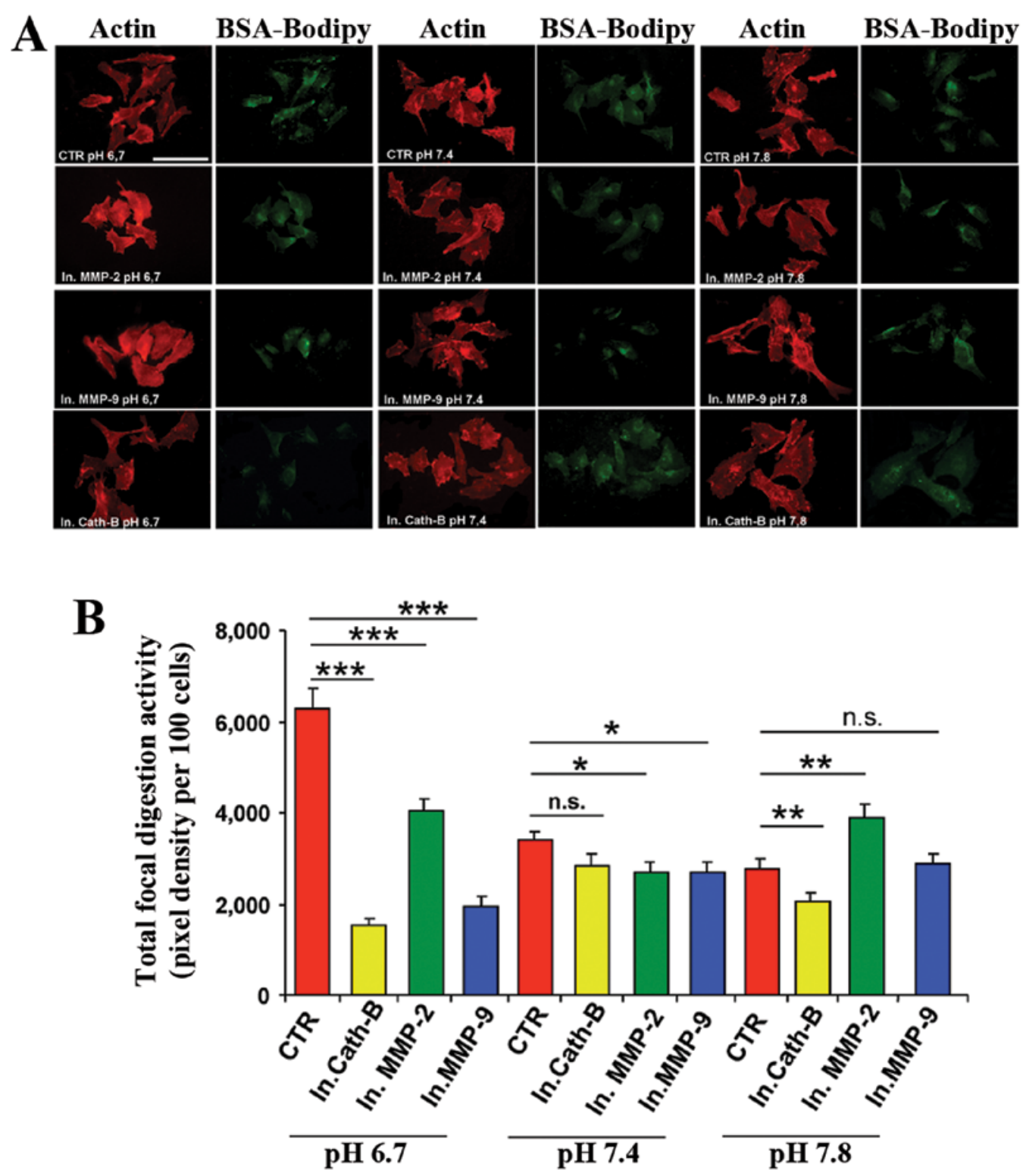

Figure 4. Inhibition of MMP-2/-9 and cathepsin B protease activity inhibits matrix degradation by the cells, particularly at acidic pH. Effect of different pHe on MMP-2/-9 and cathepsin B protease activity at invadopodia from cells cultured overnight in Matrigel/BSA-Bodipy and incubated in acidic (pHe 6.7), neutral (pHe 7.4) or basic (pHe 7.8) growth mediums, in the absence or presence of the following protease inhibitors, cathespin B inhibitor CA-074 (5 $\mu \mathrm{M})$, MMP-9 inhibitor $1(7.5 \mathrm{nM})$ or MMP-2 inhibitor $1(15 \mu \mathrm{M})$. (A) Typical fields of actin (red) stained cells and proteolysis (green) in the three $\mathrm{pHe}$ conditions. Bar=50 $\mu \mathrm{m}$. (B) Total amount of focal digestion measured in pixel density as described in Materials and methods. Results are given as means \pm SEM $(n=4) ;{ }^{*} p<0.01$ and ${ }^{* * *} \mathrm{p}<0.001$ statistically significantly different from control cells for each pHe value. MMP-2/-9, matrix metalloproteinase-2/-9; pHe, extracellular $\mathrm{pH}$.

\section{Discussion}

Matrix metalloproteases (i.e. MMP-2, -9 and MT1-MMP) and cysteine proteases such as cathepsin B are key invasion markers due to their critical role in digesting both extracellular matrix (ECM) and basement membrane proteins (25). However, except for the membrane anchored MT1-MMP, there is some controversy concerning the respective importance of each protease in ECM digestion, their interaction and the role of extracellular $\mathrm{pH}(\mathrm{pHe})$ in driving or regulating protease activity and action at the most important sites of ECM digestion by cancer cells, the invadopodia.

While a large series of observations demonstrate that NHE1-dependent acidification of the extracellular space functions together with extracellular proteases to digest the ECM in a controlled manner, the experiments were performed with whole conditioned medium; therefore, the structural and functional mechanisms/determinants linking the extracellular acidification to the ECM proteolysis remain unknown. In this context, data from various groups has shown that NHE1-dependent proton extrusion at the invadopodia site is a crucial event for localized ECM digestion $(12,26,27)$. However, while it has been demonstrated for MT1-MMP that its accumulation at mature-ECM digesting invadopodia is a $\mathrm{pH}$ dependent-event (21), the presence, $\mathrm{pH}$ dynamics and interaction with NHE1 of the other proteases at invadopodia are unknown. The goal of the present study was to elucidate other proteases present at or surrounding the invadopodia and the 
dynamics of both their regulation by $\mathrm{pHe}$ and their relationship with NHE1.

To highlight these three key processes, we applied the following experimental strategies; first, we measured the cellular expression and secretion of cathepsin B, MMP-2 and -9 in cells incubated in neutral ( $\mathrm{pHe} 7.4$ ), in acidic ( $\mathrm{pHe} 6.7$ ) or in basic (pHe 7.8) growth medium and these experiments confirmed that all three proteases are secreted at higher levels at lower pHe (Fig. 1). Furthermore, we reported for the first time in a single study by invadopodia fractionation experiments that all these proteases compartmentalize with NHE1 at invadopodia (Fig. 2A). In this respect, an important novel observation of the present study was that, in ECM-digesting invadopodia, NHE1 was very closely associated with the three proteases, such that we observed a positive interaction signal in an in situ assay (proximity ligation assay) that measures interactions between two proteins in close proximity (under $40 \mathrm{~nm}$ ) with high spatial resolution (28) (Fig. 2B). This suggests that a tight physical and functional association between the major enzyme responsible for extra-invadopodial acidification, NHE1, and the acidic-driven proteases in that microspace exists in invadopodia. Also, as NHE1 is associated with MMP-2/-9 and cathepsin B at invadopodia, we investigated whether NHE1 activity is important for the secretion of the three proteases into the peri-invadopodial space and if protease activity may possibly reciprocally regulate NHE1 expression at the invadopodia. We observed a dose-dependent stimulation of the release of cathepsin B, MMP-2 and -9 by NHE1 inhibitor, cariporide (Fig. 3B) at acidic pHe, indicating a strict control of NHE1 over the protease secretion pathway. Lastly, as a direct measure of a $\mathrm{pH}$-dependent regulation of protease activity at the invadopodia site of ECM digestion is still lacking, we performed a series of in situ zymogram experiments in the absence and presence of specific inhibitor of each protease, confirming that the major site of protease digestion of the ECM occurs at invadopodia (Fig. 4A and B). These data indicate that more than one protease is functioning to digest the ECM at invadopodia and that the proteases have a functional $\mathrm{pHe}$ optimum that is acidic not only in vitro but also in situ. Indeed, our use of small-molecule inhibitors to pharmacologically knock-out protease function revealed that each of the proteases plays important roles in invadopodia proteolysis of the ECM and, therefore, presumably in proteolysis-dependent functions such as tumor growth, tumor vascularity and invasion.

In conclusion, our data demonstrate for the first time that proton extrusion at the invadopodial site is a crucial event for proteolytic ECM digestion in that at the invadopodial sites of ECM proteolysis, more than one protease is functioning to digest the ECM and these proteases have a functional $\mathrm{pHe}$ optimum that is acidic. These data provide a structural basis for the well known role of the NHE1 in tumor cell invasion and the regulation of protease activity localized at invadopodia.

\section{Acknowledgements}

This study was supported by 'Associazione Italiana per la Ricerca sul Cancro' (AIRC) grant no. 11348 and PRIN Grant 2009 no. 1341 to S.J.R. The SJR laboratory is part of the
Italian network 'Istituto Nazionale Biostrutture e Biosistemi' (INBB), and the 'Centro di Eccellenza di Genomica in Campo Biomedico ed Agrario' of the University of Bari and the project 'BioBoP' of the Region Puglia.

\section{References}

1. Spano D and Zollo M: Tumor microenvironment: a main actor in the metastasis process. Clin Exp Metastasis 29: 381-395, 2012.

2. Cardone RA, Casavola V and Reshkin SJ: The role of disturbed $\mathrm{pH}$ dynamics and the $\mathrm{Na}^{+} / \mathrm{H}^{+}$exchanger in metastasis. Nat Rev Cancer 5: 786-795, 2005.

3. Bailey KM, Wojtkowiak JW, Hashim AI and Gillies RJ: Targeting the metabolic microenvironment of tumors. Adv Pharmacol 65: 63-107, 2012

4. Martin NK, Robey IF, Gaffney EA, Gillies RJ, Gatenby RA and Maini PK: Predicting the safety and efficacy of buffer therapy to raise tumour pHe: an integrative modelling study. $\mathrm{Br} \mathrm{J}$ Cancer 106: 1280-1287, 2012.

5. Bourguignon LY, Singleton PA, Diedrich F, Stern R and Gilad E: CD44 interaction with $\mathrm{Na}^{+}-\mathrm{H}^{+}$exchanger (NHE1) creates acidic microenvironments leading to hyaluronidase- 2 and cathepsin $\mathrm{B}$ activation and breast tumor cell invasion. J Biol Chem 279: 26991-27007, 2004.

6. Rofstad EK, Mathiesen B, Kindem K and Galappathi K: Acidic extracellular $\mathrm{pH}$ promotes experimental metastasis of human melanoma cells in athymic nude mice. Cancer Res 66: 6699-6707, 2006.

7. Estrella V, Chen T, Lloyd M, Wojtkowiak J, Cornnell HH, IbrahimHashim A, et al: Acidity generated by the tumor microenvironment drives local invasion. Cancer Res 73: 1524-1535, 2013.

8. Reshkin SJ, Cardone RA and Harguindey S: $\mathrm{Na}^{+}-\mathrm{H}^{+}$exchanger, $\mathrm{pH}$ regulation and cancer. Recent Pat Anticancer Drug Discov 8: 85-99, 2013.

9. Mason SD and Joyce JA: Proteolytic networks in cancer. Trends Cell Biol 21: 228-237, 2011.

10. Eckert MA and Yang J: Targeting invadopodia to block breast cancer metastasis. Oncotarget 2: 562-568, 2011.

11. Yamaguchi H: Pathological roles of invadopodia in cancer invasion and metastasis. Eur J Cell Biol 91: 902-907, 2012.

12. Busco G, Cardone RA, Greco MR, Bellizzi A, Colella M, Antelmi E, et al: NHE1 promotes invadopodial ECM proteolysis through acidification of the peri-invadopodial space. FASEB J 24: 3903-3915, 2010.

13. Cardone RA, Greco MR, Capulli M, Weinman EJ, Busco G, Bellizzi A, et al: NHERF1 acts as a molecular switch to program metastatic behavior and organotropism via its PDZ domains. Mol Biol Cell 23: 2028-2040, 2012.

14. Cardone RA, Bagorda A, Bellizzi A, Busco G, Guerra L, Paradiso A, et al: Protein kinase A gating of a pseudopodiallocated RhoA/ROCK/p38/NHE1 signal module regulates invasion in breast cancer cell lines. Mol Biol Cell 16: 3117-3127, 2005.

15. Roomi MW, Monterrey JC, Kalinovsky T, Rath M and Niedzwiecki A. Distinct patterns of matrix metalloproteinase-2 and -9 expression in normal human cell lines. Oncol Rep 21: 821-826, 2009.

16. Matarrese P, Ascione B, Ciarlo L, Vona R, Leonetti C, Scarsella M, et al: Cathepsin B inhibition interferes with metastatic potential of human melanoma: an in vitro and in vivo study. Mol Cancer 9: 207, 2010.

17. Brisson L, Gillet L, Calaghan S, Besson P, Le Guennec JY, Roger $\mathrm{S}$ and Gore $\mathrm{J}: \mathrm{Na}_{\mathrm{v}} 1.5$ enhances breast cancer cell invasiveness by increasing NHE1-dependent $\mathrm{H}^{+}$efflux in caveolae. Oncogene 30: 2070-2076, 2011.

18. Bowden ET, Barth M, Thomas D, Glazer RI and Mueller SC: An invasion-related complex of cortactin, paxillin and $\mathrm{PKC} \mu$ associates with invadopodia at sites of extracellular matrix degradation. Oncogene 18: 4440-4449, 1999.

19. Caldieri G, Ayala I, Attanasio F and Buccione R: Cell and molecular biology of invadopodia. Int Rev Cell Mol Biol 275: $1-34,2009$

20. Söderberg O, Gullberg M, Jarvius M, Ridderstråle K, Leuchowius KJ, Jarvius J, et al: Direct observation of individual endogenous protein complexes in situ by proximity ligation. Nat Methods 3: 995-1000, 2006.

21. Lin Y, Chang G, Wang J, Jin W, Wang L, Li H, et al: NHE1 mediates MDA-MB-231 cells invasion through the regulation of MT1-MMP. Exp Cell Res 317: 2031-2040, 2011. 
22. Putney LK and Barber DL: Expression profile of genes regulated by activity of the Na-H exchanger NHE1. BMC Genomics 5: 46, 2004.

23. Komarova SV, Pereverzev A, Shum JW, Sims SM and Dixon SJ: Convergent signaling by acidosis and receptor activator of NF- $\kappa B$ ligand (RANKL) on the calcium/calcineurin/NFAT pathway in osteoclasts. Proc Natl Acad Sci USA 102: 2643-2648, 2005.

24. Pereverzev A, Komarova SV, Korcok J, Armstrong S, Tremblay GB, Dixon SJ and Sims SM: Extracellular acidification enhances osteoclast survival through an NFAT-independent, protein kinase C-dependent pathway. Bone 42: 150-161, 2008.

25. Monet M, Lehen'kyi V, Gackiere F, Firlej V, Vandenberghe M, Roudbaraki M, et al: Role of cationic channel TRPV2 in promoting prostate cancer migration and progression to androgen resistance. Cancer Res 70: 1225-1235, 2010.
26. Lucien F, Brochu-Gaudreau K, Arsenault D, Harper K and Dubois CM: Hypoxia-induced invadopodia formation involves activation of NHE-1 by the p90 ribosomal S6 kinase (p90RSK). PLoS One 6: e28851, 2011.

27. Magalhaes MA, Larson DR, Mader CC, Bravo-Cordero JJ, Gil-Henn $\mathrm{H}$, Oser $\mathrm{M}$, et al: Cortactin phosphorylation regulates cell invasion through a $\mathrm{pH}$-dependent pathway. J Cell Biol 195: 903-920, 2011.

28. Fredriksson S, Gullberg M, Jarvius J, Olsson C, Pietras K, Gústafsdóttir SM, et al: Protein detection using proximitydependent DNA ligation assays. Nat Biotechnol 20: 473-477, 2002. 